\title{
Vehicle Routing Problem Instances: Application to Multi-UAV Mission Planning
}

\author{
Mariam Faied, Ahmed Mostafa, and Anouck Girard
}

\begin{abstract}
For many decades, the Vehicle Routing Problem (VRP) and its different variants have been studied and found applications in the real world. This paper briefly surveys VRP instances with applications to multi-objective Unmanned Aerial Vehicle (UAV) operations. Focusing on multi-objective multi-UAV mission planning problems, we try to take advantage of the literature in the VRP and its variants. We show that each military multi-UAV mission has its corresponding VRP variant. We present a novel algorithm that relies on an enhanced tree search algorithm to solve complex multi-UAV mission planning problems with complex constraints. In simulation, we introduce examples for practical problem sizes in military UAV applications.
\end{abstract}

\section{Introduction}

The VRP is faced every day by thousands of distributors worldwide and has significant economic importance. In recent years, many service suppliers and distributors have recognized the importance of designing efficient distribution strategies to improve the level of customer's service. The VRP arises naturally ${ }^{1}$ as a central problem in the fields of transportation, distribution and logistics. Some market sectors have reported that utilization of computerized methods for transportation often results in significant savings ranging from $5 \%$ to $20 \%$ in the total costs. ${ }^{2}$ In the last decade, new insights and algorithms have been obtained for the classical determinstic VRP as well as for natural stochastic and dynamic variations of it. These new developments are based on theoretical analysis, combined probabilistic and combinatorial modeling, and lead to new and effective algorithms and a deeper understanding of uncertainty issues in vehicle routing problems. ${ }^{3}$ The VRP is an NP-hard problem with many extensions such as the Vehicle Routing Problem with Time Windows (VRPTW) and the Multiple Depot Vehicle Routing Problem (MDVRP). These extensions easily lend themselves to Unmanned Aerial Vehicles (UAV) task assignment problems. Cosidering the VRP applications, military UAV missions have emerged in the literature in the last decade. ${ }^{4-6}$ The UAV routing problem is comparable to the Vehicle Routing Problem (VRP). The VRP optimizes the routes that several vehicles should follow when delivering goods to a network of customers from a single place of origin, a depot. When assigning UAVs, the customers are analogous to targets and the depot is the launch and landing site. $\mathrm{In},{ }^{7,8} \mathrm{~A}$ VRPTW is developed to minimize both the total distance of the routes and the number of vehicles by minimizing the summation of all chosen routes between customers in a supply delivery scenario. Joint and overlapping tasks and limited time windows were imposed in. ${ }^{9} \mathrm{In}^{6}{ }^{6}$ a MILP formulation for a wide area search munitions team of UAVs to search and destroy various targets was implemented. Each target required three distinct tasks to be executed in a specific order. In, ${ }^{4}$ a vehicle routing algorithm was applied to an ISR type scenario using a small team of UAVs with specific emphasis on an urban environment.

Because the UAV task assignment problem is NP-hard, the size of the problem and thus the computational effort increases exponentially. This issue is specifically investigated in. ${ }^{6}$ With five UAVs, four targets, and three tasks per target the computation was terminated because of the excessive computation time. ${ }^{6}$ A problem formulation, therefore, must be created that is robust enough to examine a wide variety of scenarios

\footnotetext{
*This work was supported by The Ministry of Higher Education in Egypt, AFRL and AFOSR under grant number FA 8650-07-2-3744.

${ }^{\dagger}$ M. Faied is with the Aerospace Engineering Departement, The University of Michigan, Ann Arbor, MI USA, and the Department of Electrical Engineering, Fayoum University, Fayoum, Egypt mfaieda@umich.edu

${ }^{\ddagger}$ A. Mostafa is with the Evara Group, Ann Arbor, MI 48103-2140 USA am.evara@gmail.com

$\S$ A. Girard is with the Department of Aerospace Engineering, The University of Michigan, Ann Arbor, MI $48109-2140$ USA anouck@umich.edu
} 
and accommodate additional operator constraints, but must also be computationally efficient. Focusing on the VRP from the military multi-UAV task assignment perspective, there is a number of disparities. In, ${ }^{7,8}$ there is only one depot since the vehicles will be routinely traveling along their assigned routes. The combat environment of the military in which the teams of UAVs will be operating will not necessarily be limited to just one depot or launch site; the scenario may require multiple different launch and landing sites. The time windows of the VRPTW will also not necessarily need to be imposed, but an operator may choose to impose various other timing constraints.

In this paper, we focus the discussion on military mission planning in which there might be heterogeneous targets and UAVs, and perhaps not all the objectives must be accomplished nor all UAVs must be used. For instance, an objective might be to cover a subset of the targets, assuming that some other criteria are satisfied, e.g. another partial task has been completed or a specified amount of time has elapsed before servicing this target. The mission planning algorithm should be able to decide when and which target to service using perhaps a cautiously selected subset of the UAVs in order not to risk them all. Given these specifications, solution methods are also required to plan the mission in an optimal way considering the given constraints. In this paper, we propose an enhanced tree search based algorithm that yields the exact solution for a broad subset of these combinatorial problems. We argue by examples that this algorithm can handle the basic VRP-like mission as well as different variations of it. We also employ a slightly generalized version of Multiple Depot Vehicle Routing Problem with Time Windows (MDVRPTW), to which we add certain technical and timing specifications, encoded as a set of linear constraints. We also present examples of practical sizes with complex mission objectives and several temporal constraints in the paper.

The rest of this paper is organized as follows. In section II we formally introduce the basic Vehicle Routing Problem definition, formulation, and classification. A survey of different VRP instances with applications to military missions is discussed in section III. Our proposed methodology to solve different complex military UAV missions is presented in section IV. Section V introduce simulation results for example missions. The paper ends with conclusion.

\section{Basic VRP}

The Vehicle Routing Problem (VRP) is one of the most challenging combinatorial optimization and nonlinear programming problems. The interest in VRP is motivated by its practical relevance as well as by its considerable difficulty. In this section, we introduce the basic VRP definition, formulation, classification, and most common solution techniques.

\section{II.A. VRP Definition}

The Vehicle Routing Problem (VRP) (problem formulation) is a generic name given to a whole class of problems in which a set of routes for a fleet of vehicles based at one or several depots must be determined for a number of geographically dispersed cities or customers. The objective of the VRP is to deliver a set of customers with known demands on minimum-cost vehicle routes originating and terminating at a depot. Numerous methods have been developed for searching the optimum solution for the problem, but even for the smallest problems, finding the global minimum for the cost function is computationally complex.

\section{II.B. VRP Formulation}

The VRP is a combinatorial problem whose ground set is the edges of a graph $G=(V, E)$. The notation used for this problem is as follows:

- $V=\left\{v_{0}, v_{1}, \ldots, v_{n}\right\}$ is a vertex set of locations.

- $E$ is the set of $n$ cities.

- $C$ is a matrix of non-negative costs or distances $c_{i j}$ between customers $v_{i}$ and $v_{j}$.

- $R_{i}$ is the route for vehicle $i$.

- $m$ is the number or vehicles (all identical and traveling at constant speeds). One route is assigned to each vehicle. 
When $c_{i j}=c_{j i}$ for all $\left(v_{i}, v_{j}\right)$ the problem is said to be symmetric. The VRP thus consists of determining a set of $m$ vehicles routes of minimal total cost, starting and ending at a depot, such that every vertex in $V$ is visited exactly once by one vehicle. The cost of a given route $\left(R i=v_{0}, v_{1}, \ldots, v_{n+1}\right)$, where $v_{i} \in V$ and $v_{0}=v_{n+1}=0$ ( 0 denotes the depot), is given by:

$$
C\left(R_{i}\right)=\sum_{i=0}^{n} c_{i, i+1}
$$

A route $R_{i}$ is feasible if the vehicle stops exactly once at each customer's location and the total duration of the route does not exceed a pre-specified bound $D: C\left(R_{i}\right) \leq D$. Finally, the cost of the problem solution is:

$$
F_{V R P}(S)=\sum_{i=1}^{m} C\left(R_{i}\right)
$$

\section{II.C. VRP Classification}

While the classical view of the VRP is static and deterministic, in many of the practical applications there are significant stochastic and dynamic components to the problem. Indeed, demands in many real life logistics systems arrive randomly in time as well as in size. Thus multiple-vehicles routing is a continuous process of collecting data, forming tours, and dispatching vehicles. The analysis of these stochastic and dynamic VRPs provides structural insight into the effect on the global routing performance of the number, speed, and capacity of vehicles employed, service region size, and the target locations distribution. For every routing instance, there is a corresponding bin-packing instance. ${ }^{3}$ Thus we can say that VRP-this difficult combinatorial problem- conceptually lies at the intersection of these two well-studied problems:

- The Traveling Salesman Problem (TSP): Assuming that the capacity of the vehicles $C$ is infinite, we can get an instance of the Multiple Traveling Salesman Problem (MTSP). A MTSP instance can be transformed into an equivalent TSP instance by adjoining to the graph $k-1$ (being $\mathrm{k}$ the number of routes) additional copies of node 0 and its incident edges.

- The Bin Packing Problem (BPP): The existence of a feasible solution for a given instance of the VRP is an instance of the BPP. The decision version of this problem is conceptually equivalent to a VRP model in which all edge costs are taken to be zero (so that all feasible solutions have the same cost).

A feasible solution to the full problem is a TSP tour (in the expanded graph) that also satisfies the packing constraints (i.e., the total demand along each of the $k$ segments joining successive copies of the depot does not exceed $C)$.

\section{II.D. Solution Techniques}

Due to the NP-Hardness of the VRP, no exact algorithm can be guaranteed to stumble on optimal tours within reasonable computing time when the number of cities is large. In, ${ }^{10}$ the K-tree method succeed in solving a problem with 71 customers for the CVRP (Capacitated VRP, defined below). To treat larger instances, or to obtain solutions faster, heuristic methods must be used. Heuristic methods perform a relatively limited exploration of the problem's search space and typically produce good quality solutions within modest computing times. ${ }^{11-13}$ In the hope of obtaining a more efficient or more robust procedure, metaheuristics have been proposed. Metaheuristics are generally applied to problems for which there is no satisfactory problem-specific algorithm or heuristic; or when it is not practical to implement such a method. In metaheuristics, the emphasis is on performing a deep exploration of the most promising regions of the solution space. The quality of solutions produced by these methods is much higher than that obtained by classical heuristics. ${ }^{14,15}$ 


\section{VRP Instances with Applications to Military Missions}

Intrinsically, the VRP is a spatial problem. Focusing on military VRPs, temporal aspects of routing problems have become increasingly important. Some of the most important instances are:

1. Capacitated VRP (CVRP): CVRP is a Vehicle Routing Problem (VRP) in which a fixed fleet of delivery vehicles of uniform capacity must service known customers demands for a single commodity from a common depot at minimum transit cost. That is, the CVRP is like the VRP with the additional constraint that every vehicle must have uniform capacity of a single commodity. ${ }^{16}$ A bomber dropping a certain number of bombs to attack ground or sea targets is a military example of this problem.

2. Multiple Depot VRP (MDVRP): A MDVRP requires the assignment of customers to depots. A fleet of vehicles is based at each depot. Each vehicle originates from one depot, services the customers assigned to that depot, and returns to the same depot. ${ }^{17}$ The objective of the problem is to service all customers while minimizing the number of vehicles and travel distance. This aspect of VRP is characteristic of military problems where the vehicles can take-off and land from one of multiple bases.

3. Stochastic VRP (SVRP): SVRPs are VRPs where one or several components of the problem are random. Three different kinds of SVRP are: Stochastic customers, Stochastic demands, and Stochastic times. ${ }^{18}$ Uncertainty in targets' locations, arrival times, or types are military examples of this type of VRP.

4. Periodic VRP (PVRP): In classical VRPs, typically the planning period is a single day. In the case of the Periodic Vehicle Routing Problem (PVRP), the classical VRP is generalized by extending the planning period to $\mathrm{M}$ days. ${ }^{19}$ In a prototypical mission in a military setting, teams of Unmanned Aerial Vehicles (UAVs) can be used for wide area surveillance or border patrol everyday. In these missions, the UAVs must ensure continued coverage of a certain area.

5. Split Delivery VRP (SDVRP): SDVRP is a relaxation of the VRP wherein it is allowed that the same customer be served by different vehicles if it reduces overall costs. This relaxation is very important if the sizes of the customer orders are as big as the capacity of a vehicle. ${ }^{20}$ This instance's military paradigm can represent a group of UAV cooperating to attack tactical targets or troop concentrations.

6. Vehicle Routing Problem with Time Windows (VRPTW): The VRPTW is the same problem as the VRP with the additional restriction that in VRPTW a time window is associated with each customer, defining an interval wherein the customer has to be supplied. The interval at the depot is called the scheduling horizon. ${ }^{21}$ Rendering a detonator inoperative before it triggers an explosive device is a conventional military example for VRPTW.

7. The Vehicle Routing Problem with Pick-up and Delivery (VRPPD): VRPPD is a VRP in which the possibility that customers return some commodities is contemplated. So in VRPPD we need to take into account that the goods that customers return to the delivery vehicle must fit into it. This restriction make the planning problem more difficult and can lead to bad utilization of the vehicles capacities, increased travel distances or a need for more vehicles. ${ }^{22}$ A good illustration for this instance is a friendly unit which is pinned down by enemy units and needs to be rescued.

Many other variants as Capacitated VRP with Time Windows (CVRPTW), ${ }^{23}$ Multiple Depot VRP with Time Windows (MDVRPTW) ${ }^{24}$ Periodic VRP with Time Windows (PVRPTW) ${ }^{25}$ and more were also studied in the literature in the last decades. 


\section{SOLUTION APROACH}

In this section, we present an algorithm that is capable of handling different VRP instances. We do this in order to emphasize real issues in multi-UAVs mission planning. We propose an enhanced tree search algorithm that minimizes the VRP's cost function, as well as satisfies the conditions that relate to its instance and additional technical specifications that relate to the UAV mission and its targets conditions.

\section{IV.A. Vehicle Routing}

The vehicle routing module is composed of two parts. The first one is a Graphical User Interface (GUI) tool that represents the technical specifications for the UAV mission, as well as the conditions related to the VRP instance that we are handling. The second part is the tree search algorithm. In which, we built the tree representing the VRP and search it to minimize the cost function while satisfying the specifications.

\section{IV.A.1. Technical Specifications Representation}

Since the main goal of this module is to emphasize how to route a team of UAVs while satisfying some technical issues related to their mission, we introduce a GUI tool. Using this GUI tool, the operator can quickly create an environment for the mission by specifying the location of targets, UAVs, and any impassable obstacles. Using Fig.1 commands, the human operator will be able to specify the mission requirements as well. For example if the mission in a certain scenario is to service either target $T_{4}$ or $T_{1}$, using the "or" command button the user will click on target $T_{4}$ then hit the "or" button and again click on target $T_{1}$. Following these steps, the Karmen simulator ${ }^{26}$ will understand the mission requirement.

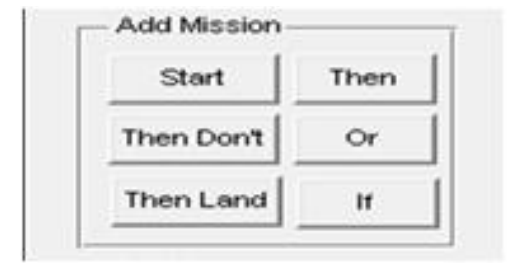

Figure 1. Specification Commands User Interface

\section{IV.A.2. Baseline Routing}

Using a tree search algorithm, this module generates a strategy for the mission that minimizes a cost function as well as satisfies the mission specifications. The cost function considered is a flight time minimization for each UAV. For minimizing the flight time, the cost used is the total distance traveled by each UAV while prosecuting the targets. Using Euclidean distance for estimating the path length of the tree branches, each UAV will have an ordered list of the targets. Other distance functions may be used as well (for example, Dubins distance).

1. Tree Representation:

For a certain number of UAVs $U=\left\{1,2, \ldots, N_{u}\right\}$ performing tasks on a set of targets $T=\left\{1,2, \ldots, N_{t}\right\}$, the tree is constructed by generating nodes that represent the assignment of a vehicle $i \in U$ to a target $j \in T$ at a specific time. The child nodes are found by enumerating all of the possible assignments that can be made, based on the remaining targets and requirements of the mission. Nodes are constructed until all combinations of vehicles and targets have been taken into account. Note that a branch of the tree, from a root node to a leaf node, represents a feasible set of targets for the UAV. If two UAVs will launch from the same site, they will have the same list of targets so they may work cooperatively on these targets. For a scenario of three vehicles servicing four targets, Fig.2 shows a part of the subtree related to $U_{1}$. In order to represent all the possible assignments for this scenario, there should be another two subtrees related to $U_{2}$ and $U_{3}$. The top node of each of these subtrees is connected to the root node of the entire tree. Each node represents one assignment of a vehicle to a target where the notation $S_{i j}$ denotes that vehicle $i$ servicing target $j$. 


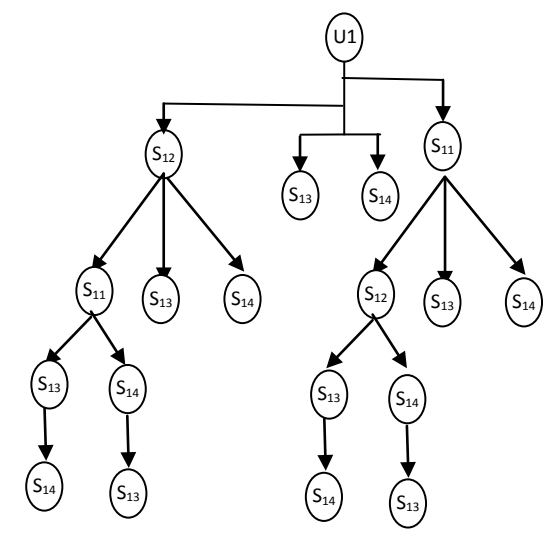

Figure 2. Sample of Feasible Assignment for One Vehicle and Four Targets

2. Search Algorithm: Our search algorithm is initiated by a Best First Search (BFS) algorithm ${ }^{27}$ that provides an immediate feasible assignment. Figure 3 presents a flow chart of the proposed BFS algorithm for our problem. It starts with the root node of the tree and the estimated cost of each child is calculated by using the lower bound Euclidean distance between the assigned vehicle and its designated target. The child node with the smallest estimate is selected and the cost of a flyable trajectory to perform the assignment is evaluated.

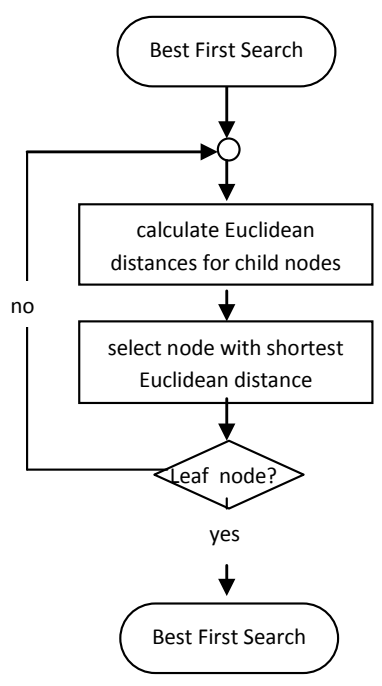

Figure 3. Best First Search Flow Chart

3. Incorporating Technical Specifications: After constructing the tree for a certain number of UAVs $N_{u}$ performing tasks on a set of targets $N_{t}$, the BFS algorithm returns the optimal flyable trajectory for this set of UAVs and targets. The simulator blocks or holds some of this set according to the mission technical specifications. The simulator returns the first set of targets the UAVs can start with now. This first set of targets includes all the targets which were entered using the Start button and targets which are not conditioned at all. All other targets will be on hold temporarily. The UAVs involved in the mission will service this set of targets according to their occurrence in the BFS trajectory. After servicing one of these Start targets, this target will either block another one "if it was entered using ThenDon't button or using Or button" or release another one "if it was entered using Then button".

4. Optimal Path: To investigate possible real-time path planning strategies of the cooperative task assignment problem in agreement with the previous conditions, it is usually assumed that the UAVs 
fly at a constant altitude and speed and we reduce the vehicle kinematics to a unicycle model with constant forward velocity. With these assumptions, the kinematic planar equations are:

$$
\begin{gathered}
\dot{x}=V \cos \psi \\
\dot{y}=V \sin \psi
\end{gathered}
$$

where $x$ and $y$ are the UAV horizontal (latitude and longitude) coordinates; $V$ is the linear velocity; and $\psi$ is the orientation. The objective is to generate a path for the UAV to follow, from a given position $\left(x_{i}, y_{i}\right)$ that ends at a given position $\left(x_{f}, y_{f}\right)$. Assuming a negligible wind condition, the UAV path must minimize the path length or the flight time - they are equal in this simplified case.

5. Performance Index: Different performance criteria can be chosen for the UAV. One performance criterion that can be used is the cumulative distance traveled by the UAVs to perform all of the required tasks,

$$
J=\sum_{i=1}^{N_{u}} D_{i} \geq 0
$$

where $D i$ is the distance traveled by UAV $i \in U$ from the beginning of the mission until finishing its part in the group task plan. The group objective is to minimize $J$ subject to the assignment requirements of the mission.

6. Combinatorial Optimization Problem: The problem is to minimize the cost function $J$ of Eq.(5) by optimizing the assignments of vehicles to targets. Let $S=\left\{1,2, . ., N_{c}\right\}$ be the set of stages in which the assignment is made. Let $x_{\{l, i, j\} \in 0,1}$ be a decision variable that is 1 if at stage $l \in S$ vehicle $i \in U$ services target $j \in T$ and is 0 otherwise. Let $c_{l, i, j}^{X_{l}-1}$ be the distance traveled by vehicle $i \in U$ to service a target $j \in T$ at a stage $l \in S$, given the prior assignment history; $r_{l, i, j}^{X_{l}-1}$ be the resource travel distance required to service a target; and $b_{i}$ be the resource availability of vehicle $i \in U$ (total distance each vehicle can travel). The mathematical formulation of the problem is:

$$
\min J=\sum_{l=1}^{N_{c}} \sum_{i=1}^{N_{u}} \sum_{j=1}^{N_{t}} c_{l, i, j}^{X_{l-1}} x_{l, i, j}
$$

Subject to

$$
\begin{array}{r}
\sum_{i=1}^{N_{u}} \sum_{j=1}^{N_{t}} x_{l, i, j}=1, l \in S ; \\
\sum_{l=1}^{N_{c}} \sum_{j=1}^{N_{t}} c_{l, i, j}^{X_{l-1}} x_{l, i, j} \leq b_{i} i \in U .
\end{array}
$$

In Eq.(6) we minimize the cost of the vehicle assignment subject to Eq.(7), which ensures that at each stage, each vehicle $i \in U$ will have exactly one target $j \in T$ assigned. Equation (8) is to limit the total resource requirement from each vehicle $i \in U$ to its capacity. After this ordered list of targets is generated, the Karmen simulator will amend it according to the mission requirements passed from the human operator. A new list of targets with their flyable trajectories will be ready for the UAV. Algorithm 1 shows the procedure for solving this problem to optimality. 


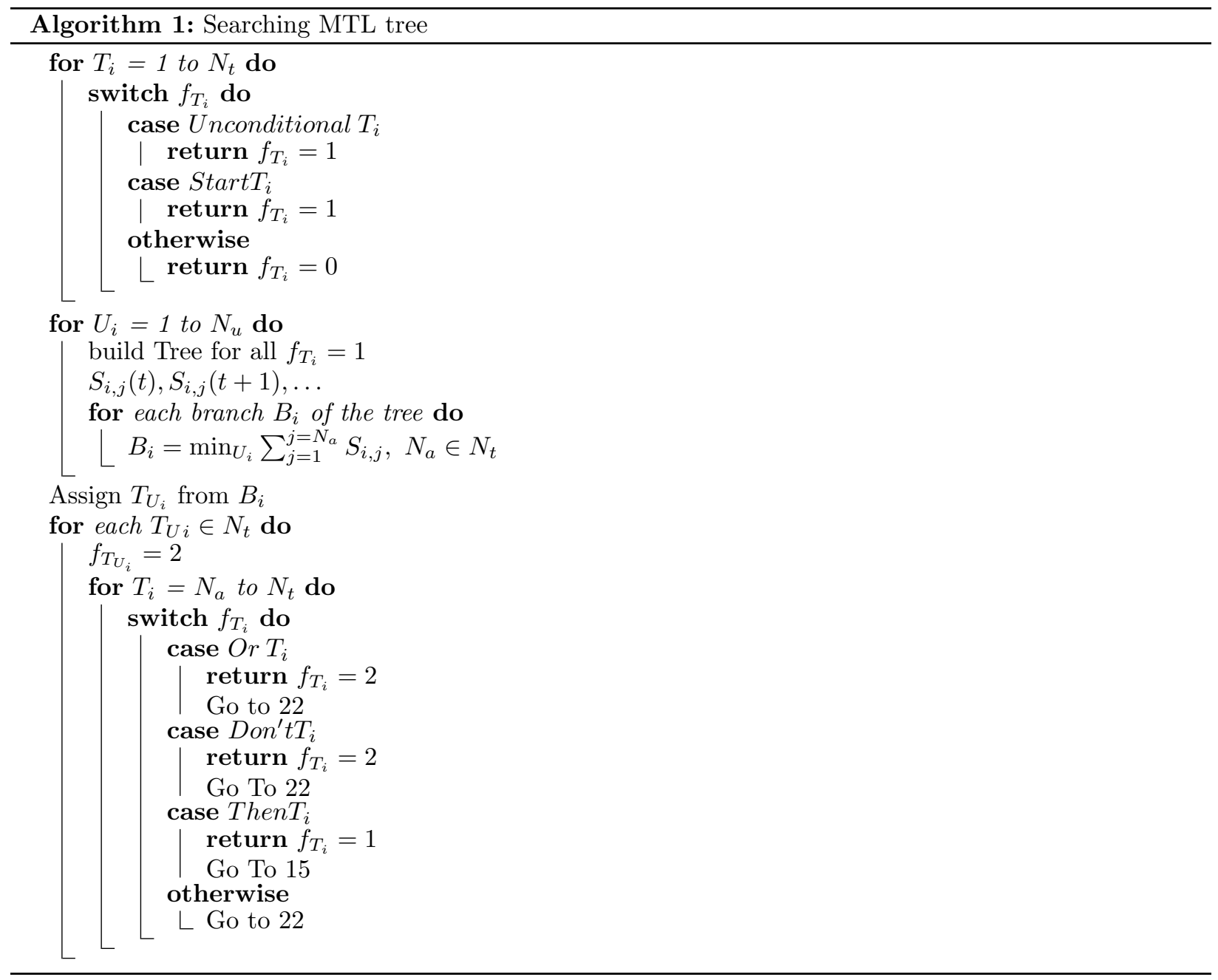

\section{SIMULATION}

In order to illustrate the framework presented in this paper, we consider three different scenarios. A first mission represents the basic VRP. UAVs with only targets with no constraints are used to illustrate our tree search algorithm. A second scenario includes different launch sites for the UAVs to represent the algorithm handling MDVRP. A third example contains multiple-UAV launch sites with some timing and technical constraints to focus on our algorithm solving the MDVRP with time windows and other technical constraints.

\section{V.A. Multi-UAV Mission as Basic VRP:}

In this mission, We have three UAVs $U_{1}, U_{2}, U_{3}$, one launch site $L_{1}$, one landing site $D_{1}$ and eight targets $T_{1}, T_{2}, T_{3}, T_{4}, T_{5}, T_{6}, T_{7}, T_{8}$. Initial conditions of targets and UAVs can be seen as in Fig. (4). In our first example, we are focusing on how our tree search algorithm works for the basic VRP. UAVs should visit targets according to the shortest path cost function order list. When calculating the time required to travel from one target to another, we used the Manhattan distance as $\mathrm{in}^{4}$ as a good measure of distance in urban settings. The total mission time was $20 \mathrm{~min}$. The servicing times for the targets were as given in table 1 . The algorithm was implemented in Matlab and run on a laptop computer equipped with a $1.8 \mathrm{GHz}$ processor and 4GB RAM. The algorithm returned the solution in about 3.5 seconds, which included the initialization time of the algorithm. 


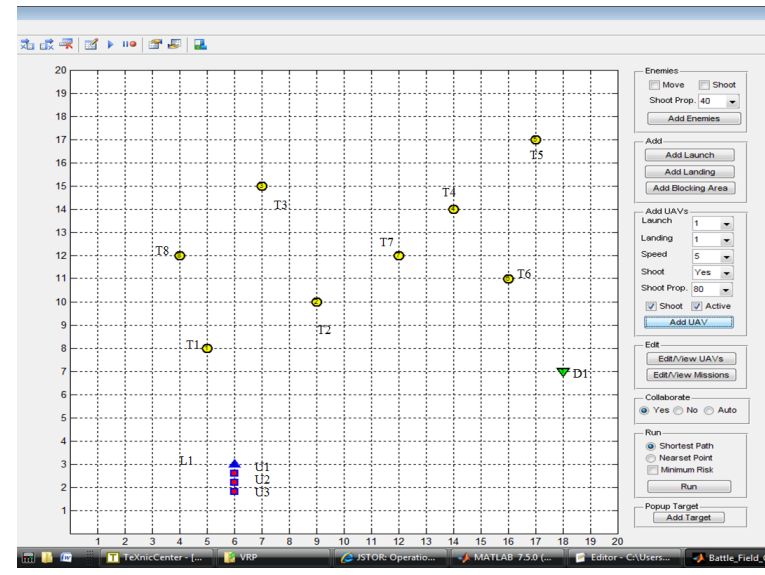

Figure 4. Military Mission A as Basic VRP

Table 1. Servicing Times For Targets in Mission A.

\begin{tabular}{ccccccccc}
\hline UAV & T1 & T2 & T3 & T4 & T5 & T6 & T7 & T8 \\
\hline U1 & 8 & 12 & & & & 17 & & \\
U2 & & & 12 & 16 & & & & \\
U3 & & & & & 19 & & 16 & 13 \\
\hline
\end{tabular}

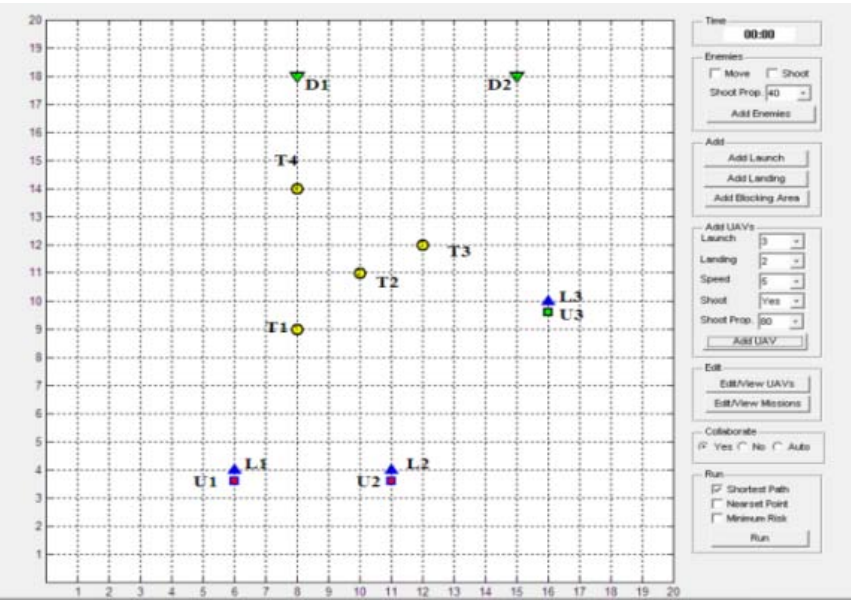

Figure 5. Map of the Military Scenario B as MDVRP

\section{9 of 11}




\section{V.B. Multi-UAV Mission as MDVRP:}

Let us consider a scenario with three UAVs, three launch sites $L_{1}, L_{2}, L_{3}$, two landing sites $D_{1}, D_{2}$ and four targets $T_{1}, T_{2}, T_{3}, T_{4}$. See Fig. 5 for the spatial distribution of the targets and the sites. The total mission time was 20 minutes. The servicing times for the targets were as given in table 2 . Notice that after $U_{2}$ serviced $T_{4}$ at 12 minutes, it traveled to land on $D_{1}$ at 20 minutes. Also $U_{1}$ serviced target $T_{1}$ and target $T_{3}$ then landed on $D_{1}$ at 17 minutes. Here, we did not involve $U_{3}$ and it will land on $D_{2}$ at 19 minutes.

Table 2. Servicing Times For Targets in Mission B.

\begin{tabular}{cllll}
\hline UAV & T1 & T2 & T3 & T4 \\
\hline U1 & 5 & & 10 & \\
U2 & & 6.5 & & 12 \\
\hline
\end{tabular}

\section{V.C. Multi-UAVs in MDVRPTW-Like Mission}

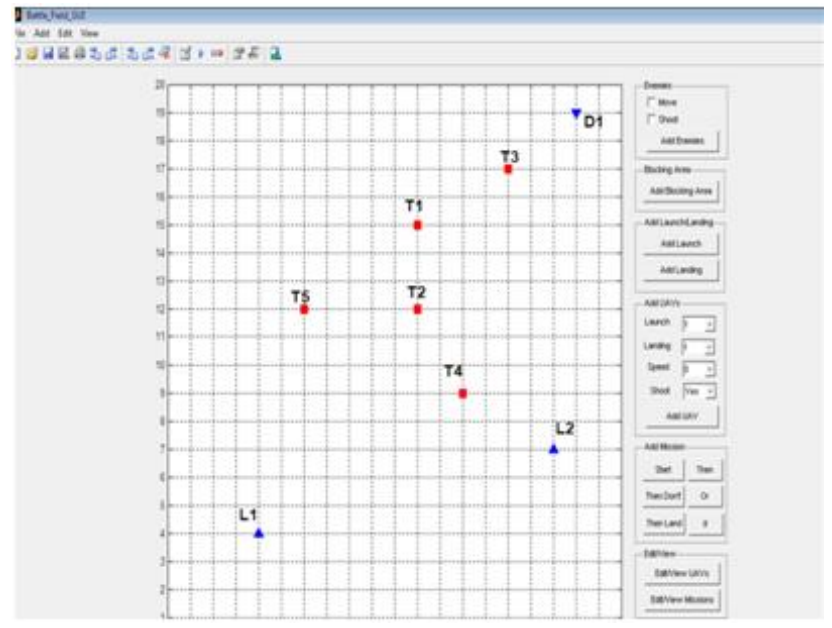

Figure 6. Map of Mission C

Let us consider another mission in order to illustrate the framework presented in this paper. Let us consider a scenario with three UAVs $U_{1}, U_{2}$, and $U_{3}$, two launch sites $L_{1}, L_{2}$, one landing site $D_{1}$, and five targets $T_{1}, T_{2}, T_{3}, T_{4}, T_{5}$. See Fig.6 for the spatial distribution of the targets and the sites. The UAVs must service targets $T_{2}, T_{3}$, and $T_{4}$ as a group within the first 45 minutes and another UAV must service targets $T_{1}$ and $T_{5}$ within 35 minutes. UAVs $U_{1}$ and $U_{3}$ are at launch site $L_{2}$ so they should be launched from there. $U_{2}$ will be launched from $L_{1}$. If $U_{1}$ takes the second option $\left(T_{1}\right.$ and $\left.T_{5}\right)$, then it must land on $D_{1}$ and it should be the last UAV to land on the landing site $D_{1}$. Furthermore, targets $T_{2}$ and $T_{3}$ must eventually be serviced by $U_{2}$ and servicing $T_{3}$ cannot be done in the first 18 minutes, but target $T_{5}$ should be serviced before $T_{3}$. After servicing $T_{3}, U_{2}$ should land on $D_{1}$ but it cannot land on $D_{1}$ until target $T_{5}$ is serviced. The mission in this case is to meet these specifications under the condition of limited flight time for each

Table 3. Servicing Times For Targets in Mission C.

\begin{tabular}{cccccc}
\hline UAV & T1 & T2 & T3 & T4 & T5 \\
\hline U1 & 32 & & & & 30 \\
U2 & & 26 & 34 & 17 & \\
\hline
\end{tabular}

UAV (60 minutes). The objective function was selected to be the total distance traveled by each UAV in 
order to minimize its flight time. The solution time of this mission was 45 minutes. The servicing times for the targets were as in table 3 . Notice that after $U_{2}$ serviced $T_{3}$ at 34 minutes, it traveled to land on $D_{1}$ at 42 minutes. Also $U_{1}$ serviced target $T_{1}$ and then landed on $D_{1}$ at 45 minutes.

\section{CONCLUSION}

In this paper, we aim to show the correlation between the VRP with its different instances and military UAV missions. Motivated mainly by military multi-UAV operations, we introduce a brief survey on the VRP instances and associate each instance with its corresponding hypothetical military mission. We aspire to take advantageous of the large literature on the VRP and its instances in the field of multi-UAV routing problems. Focusing mostly on solving complex multi-UAV mission planning problem with complex constraints, we propose an algorithm that solves the problem to optimality. The expressive power of our algorithm in handling complicated missions has been shown with three simulation examples. In the examples we attach each mission to its corresponding VRP, seeking to open the road for further papers that discuss this relationship and apply the ready made algorithms of the VRP to multi-UAV mission planning.

\section{References}

${ }^{1}$ Dantzig, G. B. and Ramser, R., "The Truck Dispatching Problem," Management Science, Vol. 6, No. 1, 1959 , pp. 8091.

${ }^{2}$ Toth, P. and Vigo, D., The Vehicle Routing Problem, SIAM, Philadelphia, 2001.

${ }^{3}$ Bertsimas, D. J. and Levi, D. S., "A New Generation of Vehicle Routing Research: Robust Algorithms, Addressing Uncertainty," Operations Research, Vol. 44, No. 2, 1996, pp. 286 - 304.

${ }^{4}$ Weinstein, A. and Schumacher, C., "UAV Scheduling via the Vehicle Routing Problem with Time Windows," Tech. Rep. AFRL-VA-WP-TP-2007-306, Air Force Research Laboratory, January.

${ }^{5}$ J. Bellingham, M. Tillerson, A. R. and J.How, Cooperative Control: Models, Applications and Algorithms, Kluwer Academic Publisher, 2001.

${ }^{6}$ Schumacher, C., Chandler, P., Pachter, M., and Pachter, L., "UAV Task Assignment with Timing Constraints via MixedInteger Linear Programming," AIAA 3rd Unmanned Unlimited Technical Conference, Workshop and Exhibit, AIAA, 2004.

${ }^{7}$ K.C.Tan, L. and K.Q.Zhu, "Heuristic Methods for Vehicle Routing Problem with Time Windows," Artificial Intelligent in Engineering, Elsevier, 2001, pp. 281-295.

${ }^{8}$ B.Ombuki, B. and F.Hanshar, "Muti-Objective Genetic Algorithms for Vehicle Routing Problem with Time Windows," Applied Intelligence, Vol. 24, 2006, pp. 17-30.

${ }^{9}$ D.Kingston and C.Schumacher, "Time-Dependent Cooperative Assignment," IEEE American Control Conference, 2005, pp. 4084-4089.

${ }^{10}$ Fisher, M. L., "Optimal Solution of Vehicle Routing Problems Using Minimum K-trees," Operations Research, Vol. 42, 1994, pp. 626-642.

${ }^{11}$ Clarke, G. and Wright, J., "Scheduling of vehicles from a central depot to a number of delivery points," Operations Research, Vol. 12, No. 4, 1964, pp. 568-581.

${ }^{12}$ D. M. Ryan, C. H. and Glover, F., "Extensions of the Petal Method for Vehicle Routing," Journal of the Operational Research Society, Vol. 44, 1993, pp. 289-296.

${ }^{13}$ Altinkemer, K. and B.Gavish, "Parallel Savings Based Heuristic for the Delivery Problem," Operations Research, Vol. 39, 1991, pp. 456-469.

${ }^{14}$ M. Zlochin, M. Birattari, N. M. and Dorigo, M., "Model-based search for combinatorial optimization: A critical survey," Operations Research, Vol. 131, 2004, pp. 373-395.

${ }^{15}$ Shaw, P., "Using Constraint Programming and Local Search Methods to Solve Vehicle Routing Problems," Fourth International Conference on Principles and Practice of Constraint Programming, 1998, pp. 417-431.

${ }^{16}$ Dorronsoro, B., "A grid-based hybrid cellular genetic algorithm for very large scale instances of the CVRP," 21ST European Conference on Modeling and Simulation ECMS 2007, 2007, pp. 759-765.

${ }^{17} \mathrm{Lim}, \mathrm{A}$. and Wang, F., "Multi-depot vehicle routing problem: a one-stage approach," IEEE Transactions on Automation Science and Engineering, Vol. 2, No. 4, 2005, pp. 397-402.

${ }^{18}$ Park, Y. B. and Hong, S. C., "A Performance Evaluation Of Vehicle Routing Heuristics In A Stochastic Environment," International Journal of Industrial Engineering: Theory, Applications and Practice, Vol. 10, No. 4, 2003, pp. 435-441.

${ }^{19}$ Angelelli, E. and Speranza, M., "The Periodic Vehicle Routing Problem with Intermediate Facilities," European Journal of Operational Research, Vol. 137, 2002, pp. 233-247.

${ }^{20}$ C. Archetti, M. S. and Speranza, M., "Worst-Case Analysis for Split Delivery Vehicle Routing Problems," Transportation Science, Vol. 40, No. 2, 2006, pp. 226-234.

${ }^{21}$ Nagata, Y., "Efficient evolutionary algorithm for the vehicle routing problem with time windows: Edge assembly crossover for the VRPTW," IEEE Congress on Evolutionary Computation, 2007, pp. 1175-1182.

${ }^{22}$ Nagy, G., "Heuristic algorithms for single and multiple depot vehicle routing problems with pickups and deliveries," European Journal of Operational Research, Vol. 1, No. 162, 2005, pp. 126-141.

${ }^{23}$ Guo-xi, L., "Application of hybrid strategies to capacitated vehicle routing problem with time windows," Industrial Engineering Journal, Vol. 1, 2006, pp. 107-119. 
${ }^{24}$ Hong, L., "A Parallel Iterative Hybrid Genetic Algorithm For Solving MDVRPTW," The Third International Conference on Computer Science Education, 2008, pp. 229-234.

${ }^{25}$ Jianfeng, Z., "A vehicle routing system to solve a periodic vehicle routing problem for a food chain in Hong Kong," National Conference on Artificial Intelligence, 2008, pp. 1763-1768.

${ }^{26}$ Faied, M., Karmen User Manual, Michigan, latest ed.

${ }^{27}$ J.Pearl, Heuristics: Intelligent Search Strategies for Computer Problem Solving, Addison-Wesley, 1984. 